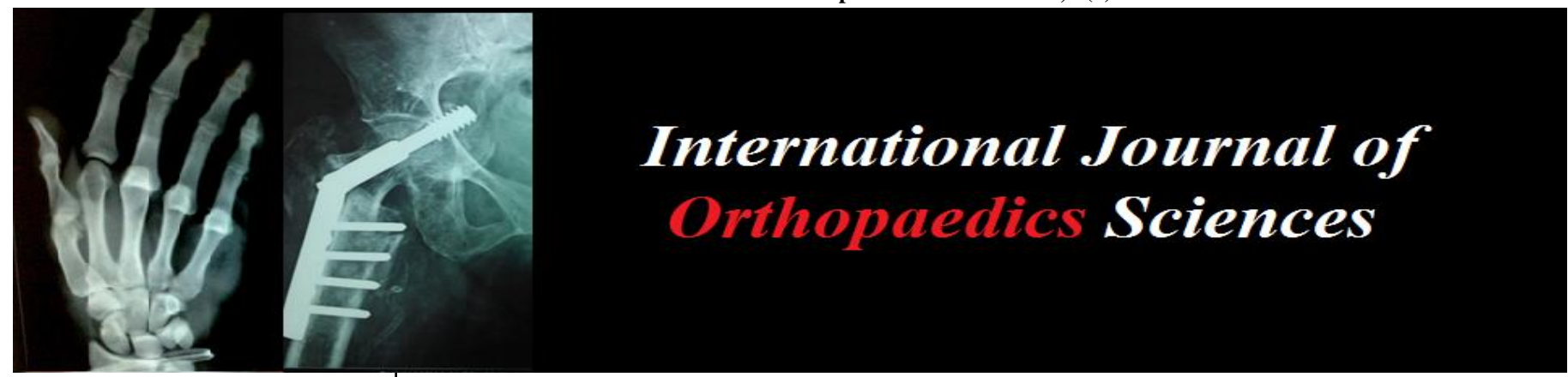

ISSN: $2395-1958$

IJOS 2019; 5(3): 642-644

(C) 2019 IJOS

www.orthopaper.com

Received: 22-05-2019

Accepted: 26-06-2019

Dr. Prashant Kumar

Senior Resident, Department of Orthopaedics, VIMS,

Ramakrishna Mission Seva

Pratishthan, Kolkata, West

Bengal, India

Prof. Dipak Kumar Jha

Professor and Head, Department of Orthopaedics, VIMS,

Ramakrishna Mission Seva

Pratishthan, Kolkata, West

Bengal, India
Correspondence

Dr. Prashant Kumar

Senior Resident, Department of

Orthopaedics, VIMS,

Ramakrishna Mission Seva

Pratishthan, Kolkata, West

Bengal, India

\section{Comparative study between platelet rich plasma and local steroid injection in tennis elbow}

\section{Dr. Prashant Kumar and Prof. Dipak Kumar Jha}

DOI: https://doi.org/10.22271/ortho.2019.v5.i3k.1605

\section{Abstract}

Tennis elbow which is due to degeneration in common origin of extensor group of muscle of forearm. In this study the comparison is done with two effective methods of management which is by giving steroid injection and platelet rich plasma (PRP). In this study two group were created of 30 patients in each group. Both the procedure was done in OPD day care basis. Patients were followed up to 6 months in gap of $1,3,6,12,24$ weeks. The outcome was measured by VAS, patient related tennis elbow evaluation (PRTEE) score and recurrence rate after 6 months.

Keywords: Tennis elbow, PRP, steroid injection

\section{Introduction}

Lateral tendinosis of the elbow popularly known as tennis elbow refers to a degenerative process in the common origin of the extensor group of muscles of the forearm ${ }^{[1]}$. PRP is promoted as an ideal autologous biological blood-derived product that can be exogenously applied to various tissues where it releases high concentrations of platelet-derived growth factors that enhance wound, bone, and tendon healing ${ }^{[2]}$. Platelets present in PRP function as a tissue sealant, initiating wound repair.

\section{Methods}

This was a prospective interventional study which includes 60 patients of all age and both genders. Patients with history of previous local injections and inflammatory arthritis were excluded from study. Patients were divided in two groups by randomisation. Local steroid injection was given to one group and platelet rich plasma (PRP) to another group. All patients were followed up to 6 months in the interval of 1,3,6,12,24 weeks.

\section{Procedure}

\subsection{Preparation of PRP}

$10 \mathrm{ml}$ blood was drawn under taking antiseptic measure and put in PRP tube, which was pyrogen free and had pre-mixed acda anticoagulant. PRP tubes are placed in centrifugation machine for the first spin of acceleration of $900 \mathrm{~g}$ for $5 \mathrm{~min}$ at 25 degrees temperature. Three layers were formed with RBC in bottom, buffy coat in middle and plasma mixed with platelet in topmost layer. Supernatant plasma containing platelets into another sterile tube (without anticoagulant) and Centrifuged at a higher speed $1500 \mathrm{~g}$ for $15 \mathrm{~min}$ to obtain a platelet concentrate. The lower $1 / 3^{\text {rd }}$ was PRP and upper $2 / 3^{\text {rd }}$ platelet-poor plasma (PPP). At the bottom of the tube, platelet pellets were formed. PPP were removed and the platelet pellets were suspended in a minimum quantity of plasma by gently shaking the tube. From this process 2-3 $\mathrm{ml}$ of PRP was prepared from $10 \mathrm{ml}$ of whole blood ${ }^{[3]}$.

$$
\mathrm{G}=1.12 * \mathrm{R} *(\mathrm{rpm} / 1000)^{2}
$$

\subsection{Method of injection}

Site was prepared in a sterile fashion with povidone-iodine solution. Using a 18-gauge needle attached to the 2-mL syringe, $2 \mathrm{ml}$ PRP or combination of $0.5 \mathrm{~mL}$ of lidocaine and $1 \mathrm{~mL}$ of 
corticosteroid (triamcinolone or betamethasone) were drawn and needle was changed to a 25-gauge. The affected arm was placed rest at the side with the elbow flexed to 45 degrees and the wrist pronated. The most tender point of the epicondyle was identified by gentle palpation. The needle was inserted at 90 degrees down to the level of the bone and then pulled back 1 to $2 \mathrm{~mm}$. aspirate it to check whether needle is in vessel. The PRP or steroid is injected at that point and needle is withdrawn. antiseptic dressing done.

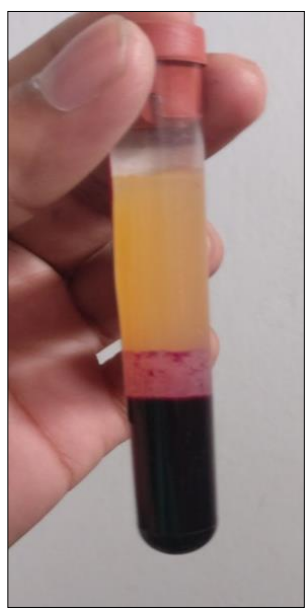

Fig 1: Layers after first spin of centrifugation. RBC in bottom, buffy coat in middle and plasma mixed platelet in top layer

\section{Results}

The mean age group of this study was 34.6 years. $62.4 \%$ were female whereas $37.6 \%$ were male. $63 \%$ of study population have right side involvement, whereas $37 \%$ affected with left side. The outcome was compared by VAS and PRTEE (patient related tennis elbow evaluation) score in subsequent visits and recurrence rate after 6 months. The mean VAS score of steroid groups in the interval of $0,1,3,6,12,24$ weeks was $8.6,4.5,3.8,3.5,2.9$ and 3.0 whereas the VAS score of PRP group was 8.8, 6.3, 5.5, 4.7, 3.2, and 2.8 respectively. The mean PRTEE score of steroid groups in the interval of $0,1,3,6,12,24$ weeks was $85.6,60.4,53.7,44.3,36.8$ and 25.2 whereas the PRTEE score of PRP group was $82.6,42.4,34.2,30.8,25.5$ and 27.1 respectively. The recurrence rate was evaluated after 6 months interval, which showed $14.6 \%$ in group of PRP injection and $28.2 \%$ recurrence in group of steroid injection. There were no neurapraxia, infection and vascular injury in this study.

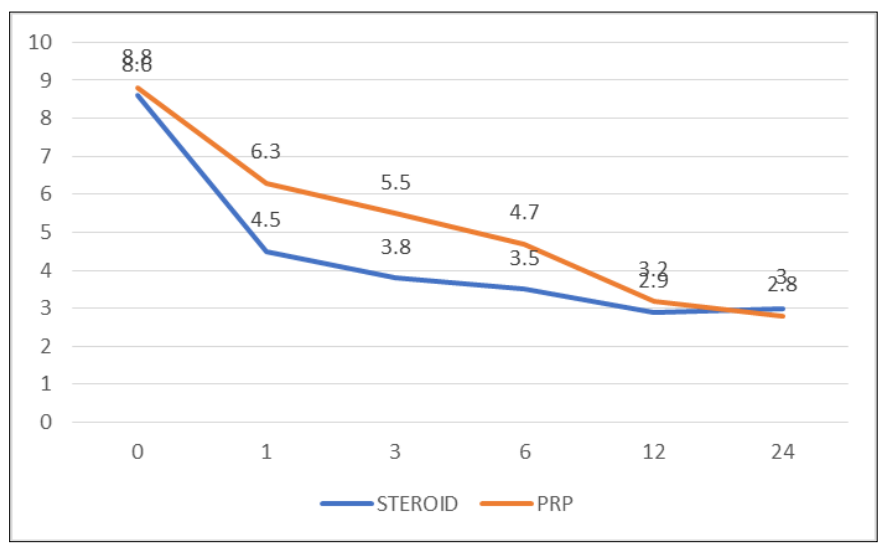

Fig 2: Vas Score

\section{Discussion}

Tennis elbow most commonly occurs in the 35-50-year age group and is more common in men than women ${ }^{[4]}$. The inflammation most commonly involves the dominant side which is right in most of the cases. The incidence is high and ever increasing and the affection is incapacitating, adversely decreasing functional ability of the person and sometimes leading to complete cessation of work activities. The treatment is still debatable with different schools of thought advocating different approaches. Rest and immobilization although seem to be the most critical part of treatment process [5]. Steroid injection has been a popular and easy method to deal this issue, but the problem of recurrence rate always compromised its effectiveness. So, a new alternative method to deal with this common problem is always under process in which PRP is one of them. In our study we found that there was female predominance with $62.4 \%$ with mainly $63 \%$ of right-side involvement. This is probably due to right hand dominance in study population. After intervention VAS score of PRP group was reduced from of $8.8 \pm 1.0$ to $2.8 \pm 1.1$ which was comparable to $7.1 \pm 1.1$ to $1.9 \pm 1.5$ in study of Martinelli et al. ${ }^{[6]}$ VAS score in steroid group fallen from $8.6 \pm 1.2$ to $3.0 \pm 1.3$ which is compared with $7.7 \pm 1.3$ to $2.8 \pm 2.1$ in study of Mohsen et al. ${ }^{[7]}$ PRTEE score in PRP group was reduced from 86.6 to 25.2 which was comparable to 79 to 23 in study by Krogh et al. ${ }^{[8]}$ The recurrence rate of steroid group $28.2 \%$ is almost double of PRP group which is $14.6 \%$ after interval of 6 months.

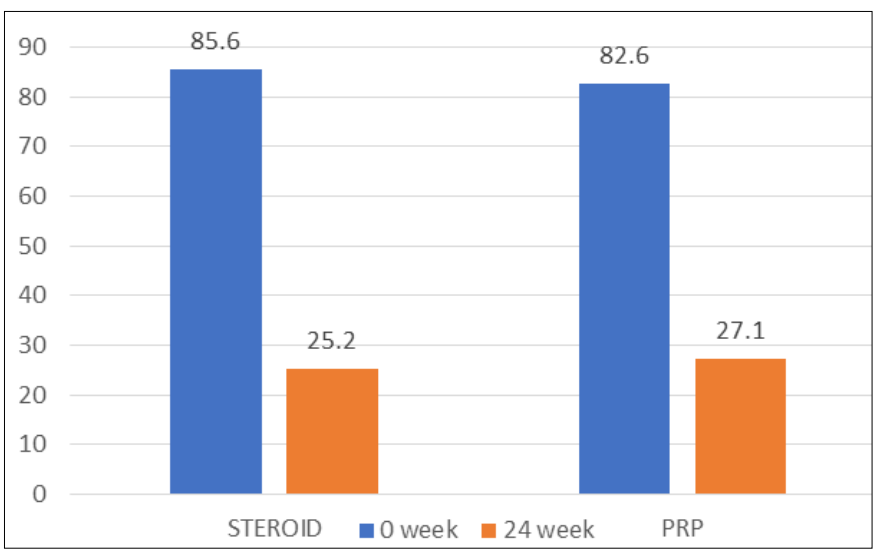

Fig 3: Prtee Score

\section{Conclusion}

Autologous PRP injection is a very promising alternative to steroid injection. While counting the advantages of PRP injection, it is safe to use, well effective to reduce pain in the long term, with low recurrence rate. It has not deleterious and serious complication of steroid injections and can also be used in diabetic patient effectively. On the contrary autologous PRP injection is costly and difficult to make while compared to steroid injection. However, a longer study with bigger sample size is needed to compare the effectiveness of PRP.

\section{Conflict of interest: No conflict of interest}

\section{References}

1. Dorf ER, Chhabra AB, Golish SR. Effect of elbow position on grip strength in the evaluation of lateral epicondylitis. J Hand Surg [Am]. 2007; 32:882-886. 10.1016/j.jhsa.2007.04.010.

2. Edwards SG, Calandruccio JH. Autologous blood injections for refractory lateral epicondylitis. J Hand Surg. 2003; 28:272-278.

3. Jo CH, Roh YH, Kim JE, Shin S, Yoon KS. Optimizing 
platelet-rich plasma gel formation by varying time and gravitational forces during centrifugation. $\mathrm{J}$ Oral Implantol. 2013; 39:525-32. Robert P. Nirschl.

4. The etiology and treatment of tennis elbow. Am J Sports Med. 1974; 2(6):308-23.

5. Esther Wagh J. Lateral Epicondylalgia or Epicondylitis: What's in a Name?. J Orthop Sports Phys Ther. 2005; 35(4):200-2.

6. Martinelli N, Marinozzi A, Carnì S et al. Platelet-rich plasma injections for chronic plantar fasciitis. Int. Orthop. 2013; 37:839-842.

7. Mardani-Kivi, Mohsen, Karimi-Mobarakeh et al. The effects of corticosteroid injection versus local anesthetic injection in the treatment of lateral epicondylitis: A randomized single-blinded clinical trial. Archives of orthopaedic and trauma surgery. 2013; 133:757-763.

8. Krogh TP, Fredberg U, Stengaard-Pedersen K, Christensen R, Jensen P, Ellingsen T. Treatment of lateral epicondylitis with platelet-rich plasma, glucocorticoid, or saline: a randomized, double-blind, placebo-controlled trial. Am J Sports Med. 2013; 41(3):625-635. 